\title{
Higher compared to lower protein diets: beneficial effects on systolic blood pressure, total cholesterol and triacylglycerol concentrations: a systematic review and meta-analysis of randomised controlled trials
}

\author{
Y.D. Vogtschmidt ${ }^{1,2}$, A. Raben ${ }^{3,4}$, I. Faber ${ }^{5}$, C. de Wilde ${ }^{6}$, J.A. Lovegrove ${ }^{1,2}$, D.I. Givens ${ }^{2}$, \\ A.F.H. Pfeiffer ${ }^{7}$ and S.S. Soedamah-Muthu ${ }^{2,6}$ \\ ${ }^{1}$ Hugh Sinclair Unit of Human Nutrition, University of Reading, Reading, UK, \\ ${ }^{2}$ Institute for Food, Nutrition and Health, University of Reading, Reading, UK, \\ ${ }^{3}$ Department of Nutrition, Exercise and Sports, Faculty of Science, University of Copenhagen, Denmark, \\ ${ }^{4}$ Steno Diabetes Center Copenhagen, Denmark, \\ ${ }^{5}$ Department of Food Science, University of Copenhagen, Denmark, \\ ${ }^{6}$ Center of Research on Psychological and Somatic disorders (CoRPS), Department of Medical and Clinical \\ Psychology, Tilburg University, The Netherlands and \\ ${ }^{7}$ Department of Endocrinology, Diabetes and Nutrition, Campus Benjamin Franklin, Charité University of Medicine, \\ Berlin, Germany
}

The benefits of a high protein diet for body weight and cardiovascular disease risk management have been reported in previous meta-analyses ${ }^{(1,2)}$. Yet, with the increasing number of recent publications on the effects of higher (HP) versus lower protein (LP) diets from randomised controlled trials (RCTs), there is a need for a renewed analysis with up-to-date evidence. The present systematic review and meta-analysis aimed to address the question of whether the totality of the current evidence from RCTs of HP versus LP diets shows beneficial effects on intermediary outcomes of cardiovascular diseases.

Systematic searches with predefined keywords were conducted in PubMed, Web of Science and Scopus, up to November 2020. RCTs, among men and women aged $\geq 18$ years, evaluating the effects of HP versus LP diets on changes in systolic (SBP) and diastolic blood pressure (DBP), total serum or plasma cholesterol, HDL- and LDL-cholesterol, and triacylglycerol concentrations were included. Three independent reviewers extracted and verified the data. Random effects meta-analyses were conducted to pool the standardised mean differences (SMDs) and 95\% confidence intervals (CIs).

Fifty-seven articles on 54 RCTs were included, involving 4,344 participants (65\% female, mean age: 46 (SD 10) years, mean body mass index (BMI): 33 (SD 3) kg/m2), with a mean study duration of 18 (range: 4 to 156) weeks. The mean protein intake of the total daily energy intake was $28 \%$ (range: 20 to $45 \%$ ) and 18\% (range: 10-23\%) in the HP and LP groups, respectively. These meta-analyses showed greater reductions in SBP (SMD -0.12, 95\% CI: $-0.21,-0.02$ from 26 trials), total cholesterol (SMD $-0.11,95 \%$ CI: $-0.19,-0.02$ from 41 trials) and triacylglycerol (SMD $-0.22,95 \%$ CI: $-0.30,-0.14$ from 43 trials), with HP compared to LP diets, with zero to very small heterogeneity (range I2: 0 to $1.3 \%$ ). This was equivalent to a greater reduction of $1.16 \mathrm{~mm} \mathrm{Hg}(95 \% \mathrm{CI}:-2.13,-0.20)$ in $\mathrm{SBP}$, $0.08 \mathrm{mmol} / \mathrm{L}$ (95\% CI: $-0.13,-0.03)$ in total cholesterol and $0.12 \mathrm{mmol} / \mathrm{L}(95 \% \mathrm{CI}:-0.16,-0.08)$ in triacylglycerol, with $\mathrm{HP}$ compared to LP diets. No significant differences were found for DBP, HDL- and LDL-cholesterol.

In these meta-analyses of 54 RCTs, we found small but beneficial effects on SBP, total cholesterol, and triacylglycerol, after higher compared to lower protein diets.

\section{Acknowledgements}

The authors thank the Rank Prize Funds, the Dutch Dairy Association and the Danish Dairy Research Foundation for funding this study and Dr Andrew Reynolds from the University of Otago, New Zealand, for his statistical advices.

\section{References}

1. Santesso N, Akl EA, Bianchi M et al. (2012) Eur J Clin Nutr 66, 780-788.

2. Wycherley TP, Moran LJ, Clifton PM et al. (2012) Am J Clin Nutr 96, 1281-1298. 